\title{
A Comparison Study of Numerical Techniques for Solving Ordinary Differential Equations Defined on a Semi-Infinite Domain Using Rational Chebyshev Functions
}

\author{
Mohamed A. Ramadan $\left(\mathbb{D},{ }^{1}\right.$ Taha Radwan $\mathbb{D},,^{2,3}$ Mahmoud A. Nassar, \\ and Mohamed A. Abd El Salam ${ }^{4}$ \\ ${ }^{1}$ Mathematics \& Computer Science Department, Faculty of Science, Menoufia University, Shebin El-Kom, Egypt \\ ${ }^{2}$ Department of Mathematics, College of Science and Arts in Ar Rass, Qassim University, Ar Rass, Saudi Arabia \\ ${ }^{3}$ Department of Mathematics and Statistics, Faculty of Management Technology and Information Systems, Port Said University, \\ Port Said, Egypt \\ ${ }^{4}$ Mathematics Department, Faculty of Science, Al-Azhar University, Nasr City, 11884 Cairo, Egypt
}

Correspondence should be addressed to Taha Radwan; t.radwan@qu.edu.sa

Received 5 October 2021; Revised 16 November 2021; Accepted 17 November 2021; Published 7 December 2021

Academic Editor: Youssri Hassan Youssri

Copyright (C) 2021 Mohamed A. Ramadan et al. This is an open access article distributed under the Creative Commons Attribution License, which permits unrestricted use, distribution, and reproduction in any medium, provided the original work is properly cited.

\begin{abstract}
A rational Chebyshev (RC) spectral collocation technique is considered in this paper to solve high-order linear ordinary differential equations (ODEs) defined on a semi-infinite domain. Two definitions of the derivative of the RC functions are introduced as operational matrices. Also, a theoretical study carried on the RC functions shows that the RC approximation has an exponential convergence. Due to the two definitions, two schemes are presented for solving the proposed linear ODEs on the semi-infinite interval with the collocation approach. According to the convergence of the RC functions at the infinity, the proposed technique deals with the boundary value problem which is defined on semi-infinite domains easily. The main goal of this paper is to present a comparison study for differential equations defined on semi-infinite intervals using the proposed two schemes. To demonstrate the validity of the comparisons, three numerical examples are provided. The obtained numerical results are compared with the exact solutions of the proposed problems.
\end{abstract}

\section{Introduction}

In the spectral methods, the most common basis functions are Chebyshev polynomials (CPs), which play an important role in the interpolation problems. Many researchers considered CPs to solve differential equations in the finite domain $[-1,1]$ (see [1-8] and [9]), but they often fail in the larger domain, also if the exact solution of the problem was in a rational form. For this reason, the rational Chebyshev (RC) functions are applied in the large domain $[0, l]$ where $l$ $\longrightarrow \infty$, which provide a major success in dealing with differential equations (DEs) defined in the open domain $[0, l]$. Many researchers studied RC functions for treating man different problems of differential, integrodifferential equations (IDEs), partial, and some other physical-engineering prob- lems as in [10,11] and [12]. Abbasbandy et al. [13] applied the RC collocation method to get numerical solution of the magnetohydrodynamic flow (MHF) of an incompressible viscous fluid (VF) over a stretching sheet problem. Ramadan et al. in [14, 15] and [16], Yalçınbas et al. in [17], and Parand and Razzaghi in [18] are scrupulous in the use of RC functions to express the approximate solution of high-order ordinary differential equations (ODEs) by different spectral approaches. In [19], Parand and Razzaghi introduced RC functions for solving a population growth of a species within a closed system, named as a Volterra model, where the authors converted the Volterra population model first to an equivalent nonlinear ODE; the solution is approximated by the RC functions with the unknown coefficients. The authors of $[20,21]$ introduced the RC function 
approximation with the collocation technique for solving the natural convection of the Darcian fluid (DF) about a vertical full cone embedded in porous media (PM) with a prescribed wall temperature. Ramadan et al. [22, 23] obtained an approximate solution of the applied collocation method based on RC functions to treat high-order linear IDEs with variable coefficients. In [24], the authors applied the RC collocation approach for approximating nonlinear biomathematical problems, namely, the systematic logistic growth, the Lotka-Volterra system (prey-predator model), the simple two-species Lotka-Volterra model, and the prey-predator model with limit cycle (periodic behavior).

All the aforementioned work either relied on the RC functions as a basis defined of an open interval or used it to treat a specific application or used truncated matrices. The truncation in matrices was handled for the first time by us in [15], and it was also an application on open period equations. In this work, a comparison study for solving linear ODEs defined on semi-infinite domains using a spectral collocation method is presented. The equation under investigation is a high-order nonhomogeneous linear variable coefficient ordinary differential equation defined on semiinfinite domain $[0, \infty)$. Two algorithms are considered by the collocation method with the RC functions as basis functions. The matrices of derivative for RC functions introduced with regular definition with truncation and improved one, which lead us to two different schemes. The collocation points that are used here are suggested by us, and they are defined on the interval $[0, \infty)$, which deal with the boundary value problems defend on $[0, \infty)$ or if one of the mixed conditions tends to infinity. So, we suggested new collocation points that are valid for dealing with a problem of this type.

\section{Preliminaries}

In this section, the definition and some properties of the RC functions are listed; also, the convergence for RC functions will be discussed.

The Chebyshev polynomials $T_{n}(x)$ are an orthonormal system in the closed interval $[-1,1]$, where the weight function for $T_{n}(x)$ is $w_{T}(x)=1 / \sqrt{1-x^{2}}$, and they may be generated using the recurrence formulae:

$$
T_{n+1}(x)=2 x T_{n}(x)-T_{n-1}(x), \quad n \geq 1,
$$

with the initials

$$
T_{0}(x)=1, T_{1}(x)=x .
$$

For more details about $T_{n}(x)$, see Ref. [8].

2.1. The RC Functions. The RC functions are orthonormal on the open interval $[0, \infty)$, defined as

$$
R_{n}(\chi)=T_{n}\left(\frac{x-1}{x+1}\right), \quad x=\cos \varphi, \varphi \in[0, \pi],
$$

to the weight function $w_{R}(x)=x^{-1 / 2}(x+1)^{-1}$, and they can be generated with the aid of the following recurrence formulae:

$$
R_{n+1}(x)=2\left(\frac{x-1}{x+1}\right) R_{n}(x)-R_{n-1}(x), \quad n \geq 1,
$$

with the initials

$$
R_{0}(x)=1, R_{1}(x)=\frac{x-1}{x+1},
$$

and the property of the orthogonality is

$$
\int_{0}^{\infty} R_{r}(x) R_{s}(x) w_{R}(x) d x=\frac{c_{s} \pi}{2} \delta_{r s}
$$

where $c_{0}=2, c_{s}=1$ for all $s \geq 1$ and $\delta_{r s}$ is the Kronecker delta function.

Let $\Omega=\{x: 0 \leq x<\infty\}$, and we note that $R_{n}(x)$ is the eigen function of the singular Sturm-Liouville problem of the following form:

$$
w_{R}^{-1}(x) \frac{d}{d x}\left[w_{R}^{-1}(x) \frac{d}{d x} R_{n}(x)\right]+n^{2} R_{n}(x)=0, \quad x \in \Omega .
$$

2.2. Function Spaces. In this subsection, the order of convergence for RC functions will be discussed; let us begin with assuming that

$$
L_{w}^{2}(\Omega)=\left\{\xi:\|\xi\|_{w}=\left(\int_{0}^{\infty}|\xi(x)|^{2} w_{R}(x) d x\right)^{1 / 2}<\infty\right\},
$$

represent the space functions, and the inner product is denoted here as

$$
\langle\alpha, \beta\rangle_{w_{R}}
$$

such that

$$
\langle\varphi, \varphi\rangle_{w}=\left(\|\varphi\|_{w_{R}}\right)^{2} .
$$

Subsequently, from the property of orthogonality (relation (6)), we get the fact that RC functions form a set of orthonormal basis of $L_{w}^{2}(\Omega)$. Also, let us define the normed spaces $H_{w}^{r}(\Omega)$ and $H_{w, A}^{r}(\Omega)$ as

$$
\begin{gathered}
H_{w_{R}}^{r}(\Omega)=\left\{\xi:\|\xi\|_{r, w_{R}}=\left(\sum_{k=0}^{r}\left\|\frac{d^{k}}{d x^{k}} \xi\right\|_{w_{R}}\right)^{1 / 2}<\infty\right\}, \\
H_{w_{R}, \theta}^{r}(\Omega)=\left\{\xi:\|\xi\|_{r, w_{R}, \theta}=\left(\sum_{k=0}^{r}\left\|(x+1)^{r / 2+k} \frac{d^{k}}{d x^{k}} \xi\right\|_{w_{R}}\right)^{1 / 2}<\infty\right\},
\end{gathered}
$$

and they form an orthonormal set of functions with respect 
where $r \geq 0$ and $k$ is a positive integer constant, and we let $\theta$ be the Sturm-Liouville operator in (7), namely, it may be written as

$$
\theta \xi=-w_{R}^{-1}\left(w_{R}^{-1} \xi^{\prime}\right)^{\prime}
$$

Let $N$ be a positive integer such that $N<\infty$, and $\Re_{N}=$ $\operatorname{span}\left\{R_{0}, R_{1}, \cdots, R_{N}\right\}$.

Theorem 1. For any $r \geq 0$, and $c$ is a generic positive constant independent of any variable, and $\phi \in \mathfrak{R}_{N}$, then

$$
\|\xi\|_{r, w_{R}} \leq c N^{2 r}\|\xi\|_{w_{R}} .
$$

The proof of Theorem 1 is given in [25].

Since the set of RC functions is orthonormal and a complete set, we assume that $f(x)$ is defined over the space $\Omega$; then, it be may expanded in terms of RC functions as

$$
f(x)=\sum_{i=0}^{\infty} a_{i} R_{i}(x)
$$

where

$$
a_{i}=\frac{\left\langle f, R_{i}\right\rangle_{w_{R}}}{\left(\left\|R_{i}\right\|_{w_{R}}\right)^{2}}=\frac{2}{c_{s} \pi} \int_{0}^{\infty} f(x) R_{i}(x) w_{R}(x) d x .
$$

Infinite series expression (14) represents as a spectral truncated approximation as follows

$$
f_{N}(x)=\sum_{i=0}^{N} a_{i} R_{i}(x)
$$

The order of convergence for the RC function approximation will be obtained using several orthonormal projections. From (16), it is clear that $f_{N}$ is the orthogonal projection of $f$ onto $\Re_{N}$ with respect to the inner product (10). For all of the above, especially Theorem 1, the following theorem is presented and contains the order of convergence of RC functions.

Theorem 2. For any function $f$ such that $f \in H_{w_{R}, \theta}^{r}(\Omega)$, where $r \geq 0$, there exists a positive constant $c$ independent of $N$ such that

$$
\left\|f_{N}-f\right\|_{r, w_{R}} \leq c N^{-r}\|f\|_{r, w_{R}, \theta} .
$$

The complete proof of Theorem 2 is found in [13] (or see Ref. [25] for more details); this theorem shows that the RC approximation has exponential convergence.

2.3. Operational Matrix. This subsection introduces the form of operational matrix for the RC functions; the derivative of the vector $\mathbf{R}(x)=\left[R_{0}(x) R_{1}(x) R_{2}(x) \cdots R_{N}(x)\right] \quad$ can $\quad$ be expressed by

$$
\mathbf{R}^{\prime}(x)=\frac{d \mathbf{R}(x)}{d x} \approx \mathbf{R}(x) \mathbf{D}^{T},
$$

where $\mathbf{D}$ is $(N+1) \times(N+1)$ operational differentiation matrix. The elements of $\mathbf{D}$ are found by differentiating (4) and using $R_{1}(x)=(x-1) /(x+1)$, then

$$
R_{n+1}^{\prime}(x)=2\left(R_{1}(x) \cdot R_{n}(x)\right)^{\prime}-R_{n-1}^{\prime}(x),
$$

also using the multiplication relation:

$$
R_{m}(x) \cdot R_{n}(x)=\frac{1}{2}\left[R_{m+n}+R_{|m-n|}\right] .
$$

The approximation sign in (18) made by a truncation to the last column of $\mathbf{D}$ (by consideration that $R_{i}^{\prime}(x)=0$, for $i$ $>N$ ) to get an invertible square operational matrix $\mathbf{D}$ (see Ref. [14]). The structure of the matrix $\mathbf{D}$ is obtained as a lower-Heisenberg matrix. The matrix $\mathbf{D}$ can be expressed as $\mathbf{D}=\mathbf{D}_{1}+\mathbf{D}_{2}$, where $\mathbf{D}_{1}$ is a tridiagonal matrix which is obtained from

$\mathbf{D}_{1}=\operatorname{diag} \cdot\left(\frac{7}{4}(i-1),-(i-1), \frac{1}{4}(i-1)\right), \quad i=1,2, \cdots, N+1$,

and the entire elements of matrix $\mathbf{D}_{2}$ are $d_{i j}$, obtained from

$$
d_{21}=-1, d_{i j}= \begin{cases}0, & j \geq i-1, \\ k(i-1) c_{j}, & j<i-1 .\end{cases}
$$

In addition to $k=(-1)^{i+j+1}, c_{1}=1$ and $c_{j}=2$ for $j \geq 2$.

Consequently, the $k^{\text {th }}$-order derivative of the row matrix $\mathbf{R}(x)$, which is given in (18), is obtained as

$$
\frac{d^{k} \mathbf{R}(x)}{d x^{k}}=[\mathbf{R}(x)]^{(k)} \approx \mathbf{R}(x)\left(\mathbf{D}^{T}\right)^{k},
$$

And we note here that $\mathbf{R}^{(0)}(x)=\mathbf{R}(x), R_{i}^{\prime}(x)=0$, for $i>N$ . Definitions (18) and (23) were introduced for the first time in $[18,19]$, and many works have used them; see, for example, Refs. [20-22], [23, 24], and [17].

\section{The Improved Differentiation of the RC Functions}

In the present section, an improved definition of differentiation for the RC functions is introduced. There was a need to find an improvement to definition (23), because in the higher derivatives, when using this definition, a week approximation is obtained. The truncated definition (18) and the $k^{\text {th }}$-order derivative (23) give us a regular truncated differentiation of the RC functions (RRC). Generally, the derivative of the rational or fractional functions increases the order of the denominator (in contrast to polynomials that reduce the order at differentiation), so the truncation 
increases as the order of the derivative increases (more than one column in Equation (18)). For example, the fourthorder derivative of the vector $\mathbf{R}(x)$ at $N=3$, the truncated terms in the row vector $\mathbf{R}(x)$ are (the last four terms) $R_{4}$, $R_{5}, R_{6}, R_{7}$. This will lead to unsatisfied approximating in high-order DEs using the presented RRC definition (18). Therefore, an improved definition of the derivative of RC functions will be proposed next.

First, a vector will be inserted into (18) to treat the truncated terms, which will improve the regular definition. This manner will be called an improved derivative of the RC functions and will be indicated by IRC.

Additionally, the first-order derivative of the row matrix $R(x)$ is

$$
\mathbf{R}^{\prime}(x)=\frac{d \mathbf{R}(x)}{d x}=\mathbf{R}(x) \mathbf{D}^{T}+\mathbf{Z}(x)
$$

where

$$
\mathbf{Z}(x)=\left[\begin{array}{llllll}
0 & 0 & 0 & \cdots & 0 & d_{N+1, N+1} R_{N+1}(x)
\end{array}\right]_{1 \times(N+1)} .
$$

Theorem 3. The $k^{\text {th }}$ derivative of the matrix vector $\mathbf{R}(x)$, in terms of itself, is defined as

$$
[\mathbf{R}(x)]^{(k)}=\mathbf{R}(x)\left(\mathbf{D}^{T}\right)^{k}+\sum_{i=0}^{k-1} \mathbf{Z}^{(i)}(x)\left(\mathbf{D}^{T}\right)^{k-i-1}, \quad k \geq 1,
$$

where

$$
\mathbf{Z}^{(k)}(x)=\left[\begin{array}{llllll}
0 & 0 & 0 & \cdots & 0 & d_{N+1, N+2} \mathbf{R}_{N+1}^{(k)}(x)
\end{array}\right] .
$$

Proof. Using the assumption (24) as the first derivative, and differentiating (24), then we get

$$
\mathbf{R}^{\prime \prime}(x)=\mathbf{R}^{\prime}(x) \mathbf{D}^{T}+\mathbf{Z}^{\prime}(x)
$$

or

$$
\begin{aligned}
\mathbf{R}^{\prime \prime}(x) & =\left\{\mathbf{R}(x) \mathbf{D}^{T}+\mathbf{Z}(x)\right\} \mathbf{D}^{T}+\mathbf{Z}^{\prime}(x) \\
& =\mathbf{R}(x)\left(\mathbf{D}^{T}\right)^{2}+\mathbf{Z}(x) \mathbf{D}^{T}+\mathbf{Z}^{\prime}(x),
\end{aligned}
$$

and by induction, we get the $k^{\text {th }}$ derivative as relation (26). $\square$

As a special case, if $\mathbf{Z}(x)=0$, it leads us to the regular RC definition in relations (18) and (23). The introduced definition (24) and the $k^{\text {th }}$-order derivative (26) give us an improved differentiation of the $\mathrm{RC}$ functions.

\section{Problem Statement}

In this study, the form of high-order ODEs which represents a linear nonhomogeneous with variable coefficients defined on a semi-infinite domain is

$$
\sum_{k=0}^{m} Q_{k}(x) f^{(k)}(x)=g(x), \quad 0 \leq x<\infty
$$

which forms $m^{\text {th }}$-order ODEs; the previous forms of DEs are subjected to the following conditions:

$$
f^{(k)}\left(\gamma_{i}\right)=\lambda_{i}, \quad 0 \leq \gamma_{i}<\infty, i=0,1, \cdots, m-1,
$$

where the $Q_{k}(x)$ and $g(x)$ are well-defined functions on $\Omega$, and $\gamma_{j}$ and $\lambda_{i}$ are constants (initial value problem), where $\gamma_{j}$ may tend to $\infty$ (boundary value problems).

Now, we consider that the approximate solution $f_{N}(x)$ according to (16) for the exact solution $f(x)$ of equation (30) in the vector form as

$$
f_{N}(x)=\sum_{n=0}^{N} a_{n} R_{n}(x)=\mathbf{R}(x) \mathbf{A}
$$

$$
f_{N}^{(k)}(x)=\sum_{n=0}^{N} a_{n}\left(R_{n}(x)\right)^{(k)}=\mathbf{R}^{(k)}(x) \mathbf{A}
$$

where

$$
\mathbf{A}=\left[\begin{array}{llll}
a_{0} & a_{1} & \cdots & a_{N}
\end{array}\right]
$$

\section{Fundamental Relation-Based Matrix Forms}

In the beginning, we provide the fundamental matrix relation of the solution of (30) by two schemes using the RC collocation approach.

Assuming that the solution $f(x)$ of (30) can be expressed as relation (32), which is a truncated RC series, then $f(x)$ and its $k^{\text {th }}$ derivative $f^{(k)}(x)$ are written in the matrix forms (32) and (33) such that $k=0,1, \cdots, m$, and $m \leq N$, where $f^{(0)}(x) \equiv f(x), a_{0}, a_{1}, \cdots, a_{N}$ are the RC coefficients to be determined later.

Now, let the collocation points $x_{s}$ as

$$
x_{s}=\frac{1+\cos (s \pi / N)}{1-\cos (s \pi / N)}, \quad s=0,1, \cdots, N
$$

and at the end points $(s=0, s=N) x_{0} \longrightarrow \infty, x_{N}=0$, namely,

$$
\begin{gathered}
R_{n}(x)=1 \text { when } x \longrightarrow \infty \text {, for all } n, \\
R_{n}(x)=\cos (n \pi)=(-1)^{n} \text { when } x \longrightarrow 0, \text { for all } n .
\end{gathered}
$$

Permanently, the RC functions are convergent at both end points 0 and $\infty$; in addition, the presence of infinity in the collocation points $\left(x_{0} \longrightarrow \infty\right)$ does not cause a failure in the substitution.

Hence, upon substituting these points (35) into (30), one obtains

$$
\sum_{k=0}^{m} Q_{k}\left(x_{s}\right) f^{(k)}\left(x_{s}\right)=g\left(x_{s}\right), \quad s=0,1,2, \cdots, N
$$


The matrix form of the obtained system (37) is written farther as

$$
\sum_{k=0}^{m} \mathbf{Q}_{k} \mathbf{F}^{(k)}=\mathbf{G}
$$

where

$$
\begin{gathered}
\mathbf{Q}_{k}=\left[\begin{array}{cccc}
Q_{k}\left(x_{0}\right) & 0 & \cdots & 0 \\
0 & Q_{k}\left(x_{1}\right) & \cdots & 0 \\
0 & 0 & \ddots & \vdots \\
0 & 0 & \cdots & Q_{k}\left(x_{N}\right)
\end{array}\right], \\
\mathbf{F}^{(k)}=\left[\begin{array}{c}
f^{(k)}\left(x_{0}\right) \\
f^{(k)}\left(x_{1}\right) \\
\vdots \\
f^{(k)}\left(x_{N}\right)
\end{array}\right], \quad \mathbf{G}=\left[\begin{array}{c}
g\left(x_{0}\right) \\
g\left(x_{1}\right) \\
\vdots \\
g\left(x_{N}\right)
\end{array}\right] .
\end{gathered}
$$

5.1. The RRC Scheme. From (23), we know that the first scheme RRC gives us a derivative of RC functions from the $k^{\text {th }}$ order; thus, equation (38) takes the form

$$
\mathbf{F}^{(k)}=\mathbf{R}\left(\mathbf{D}^{T}\right)^{k} \mathbf{A},
$$

where

$$
\mathbf{R}=\left[\begin{array}{c}
\mathbf{R}\left(x_{0}\right) \\
\mathbf{R}\left(x_{1}\right) \\
\mathbf{R}\left(x_{2}\right) \\
\vdots \\
\mathbf{R}\left(x_{N}\right)
\end{array}\right]=\left[\begin{array}{cccc}
R_{0}\left(x_{0}\right) & R_{1}\left(x_{0}\right) & \cdots & R_{N}\left(x_{0}\right) \\
R_{0}\left(x_{1}\right) & R_{1}\left(x_{1}\right) & \cdots & R_{N}\left(x_{1}\right) \\
R_{0}\left(x_{2}\right) & R_{0}\left(x_{2}\right) & \cdots & R_{0}\left(x_{2}\right) \\
\vdots & \vdots & \ddots & \vdots \\
R_{0}\left(x_{N}\right) & R_{1}\left(x_{N}\right) & \cdots & R_{N}\left(x_{N}\right)
\end{array}\right] .
$$

Hence, from (38) and (40), one obtains the fundamental matrix equation for (30) as

$$
\sum_{k=0}^{m} \mathbf{Q}_{k} \mathbf{R}\left(\mathbf{D}^{T}\right)^{k} \mathbf{A}=\mathbf{G}
$$

Also, we obtain the matrix forms corresponding to condition (31) as follows: setting $x=\gamma_{j}$ in (33), we get the fundamental matrix form corresponding to the condition (31):

$$
\mathbf{R}\left(\gamma_{i}\right)\left(\mathbf{D}^{T}\right)^{k} \mathbf{A}=\lambda_{i}, \quad 0 \leq \gamma_{i}<\infty, i=0,1, \cdots, m-1 .
$$

5.2. The IRC Scheme. We studied the improved and regular differentiating $\mathrm{RC}$ functions in the preceding section. Now, we deduce the fundamental matrix relation by the IRC scheme.

Substituting relation (26) into (40), we get

$$
\mathbf{F}^{(k)}=\left\{\mathbf{R}\left(\mathbf{D}^{T}\right)^{k}+\sum_{i=0}^{k-1} \mathbf{Z}^{(i)}\left(\mathbf{D}^{T}\right)^{k-i-1}\right\} \mathbf{A},
$$

where

$$
\mathbf{Z}=\left[\begin{array}{c}
\mathbf{Z}\left(x_{0}\right) \\
\mathbf{Z}\left(x_{1}\right) \\
\vdots \\
\mathbf{Z}\left(x_{N}\right)
\end{array}\right]=\left[\begin{array}{cccc}
Z_{0}\left(x_{0}\right) & Z_{1}\left(x_{0}\right) & \cdots & Z_{N}\left(x_{0}\right) \\
Z_{0}\left(x_{1}\right) & Z_{1}\left(x_{1}\right) & \cdots & Z_{N}\left(x_{1}\right) \\
\vdots & \vdots & \ddots & \vdots \\
Z_{0}\left(x_{N}\right) & Z_{1}\left(x_{N}\right) & \cdots & Z_{N}\left(x_{N}\right)
\end{array}\right]
$$

Hence, from (38) and (44), the fundamental matrix equation for (30) is obtained as

$$
\sum_{k=0}^{m} \mathbf{Q}_{k}\left\{\mathbf{R}\left(\mathbf{D}^{T}\right)^{k}+\sum_{i=0}^{k-1} \mathbf{Z}^{(i)}\left(\mathbf{D}^{T}\right)^{k-i-1}\right\} \mathbf{A}=\mathbf{G} .
$$

Similarly, the matrix form corresponding to the condition (31) using (26) is obtained as

$$
\left\{\mathbf{R}\left(\gamma_{i}\right)\left(\mathbf{D}^{T}\right)^{k}+\sum_{i=0}^{k-1} \mathbf{Z}^{(i)}\left(\gamma_{i}\right)\left(\mathbf{D}^{T}\right)^{k-i-1}\right\} \mathbf{A}=\lambda_{i}
$$

for $i=0,1, \cdots, m-1$, so that $0 \leq \gamma_{j}<\infty$.

\section{Method of Solution}

Due to the collocation method, the regular (42) and the improved (46) fundamental matrix equations for the proposed problem (30) correspond to a system of algebraic equations with $(N+1)$ equations for the $(N+1)$ unknown RC coefficients $a_{0}, a_{1}, \cdots, a_{N} \cdot$

One writes matrix equations (42) and (46) compactly as

$$
\mathbf{S A}=\mathbf{G},
$$

or in the augmented form as

$$
[\mathbf{S} ; \mathbf{G}]
$$

Equations (43) and (47) obtain the matrix form for the condition (31); also, they are written compactly as

$$
\mathbf{H}_{i} \mathbf{A}=\left[\lambda_{i}\right]
$$

so that $\mathbf{S}$ and $\mathbf{H}_{i}$ for RRC are defined by

$$
\begin{gathered}
\mathbf{S}=\left[s_{p q}\right]=\sum_{k=0}^{m} \mathbf{Q}_{k} \mathbf{R}\left(\mathbf{D}^{T}\right)^{k}, \quad p, q=0,1, \cdots, N, \\
\mathbf{H}_{i}=\left[\begin{array}{llll}
h_{i 0} & h_{i 1} & \cdots & h_{\mathrm{iN}}
\end{array}\right]=\mathbf{R}\left(\gamma_{j}\right)\left(\mathbf{D}^{T}\right)^{k},
\end{gathered}
$$


while $\mathbf{S}$ and $\mathbf{H}_{i}$ for IRC are defined by

$$
\begin{aligned}
\mathbf{S}= & {\left[s_{p q}\right]=\sum_{k=0}^{m} \mathbf{Q}_{k}\left\{\mathbf{R}\left(\mathbf{D}^{T}\right)^{k}+\sum_{i=0}^{k-1} \mathbf{Z}^{(i)}\left(\mathbf{D}^{T}\right)^{k-i-1}\right\}, } \\
\mathbf{H}_{i}= & {\left[\begin{array}{llll}
h_{i 0} & h_{i 1} & \cdots & h_{\mathrm{iN}}
\end{array}\right] } \\
& =\left\{\mathbf{R}\left(\gamma_{j}\right)\left(\mathbf{D}^{T}\right)^{k}+\sum_{i=0}^{k-1} \mathbf{Z}^{(i)}\left(\gamma_{j}\right)\left(\mathbf{D}^{T}\right)^{k-i-1}\right\} .
\end{aligned}
$$

Hence, the approximate solution of (30) under the condition (31) may be obtained by exchanging the rows of matrices (50) by the last (or first) $m$ rows of the matrix (49), then getting the required augmented matrix as

$$
[\tilde{S} ; \tilde{\mathbf{G}}]=\left[\begin{array}{cccccc}
s_{00} & s_{01} & \cdots & s_{0 N} & ; & g\left(x_{0}\right) \\
s_{10} & s_{11} & \cdots & s_{1 N} & ; & g\left(x_{1}\right) \\
\cdots & \cdots & \cdots & \ldots & ; & \cdots \\
s_{N-m, 0} & s_{N-m, 1} & \cdots & s_{N-m, N} & ; & g\left(x_{N-m}\right) \\
h_{00} & h_{01} & \cdots & h_{0 N} & ; & \lambda_{0} \\
h_{10} & h_{11} & \cdots & h_{1 N} & ; & \lambda_{1} \\
\ldots & \ldots & \cdots & \cdots & ; & \cdots \\
h_{m-1,0} & h_{m-1,1} & \cdots & h_{m-1, N} & ; & \lambda_{m-1}
\end{array}\right] .
$$

If $\operatorname{rank} \tilde{\mathbf{S}}$ is equal to $\operatorname{rank}[\tilde{\mathbf{S}} ; \tilde{\mathbf{G}}]$, then the algebraic system has a solution, and if the two ranks are equal to $N+1$, then the solution is unique, the inverse matrix method is used here to solve the system, and one may write the matrix equation (49) as

$$
\mathbf{A}=(\tilde{\mathbf{S}})^{-1} \tilde{\mathbf{G}}
$$

Therefore, the RC coefficients $a_{n}, n=0,1, \cdots, N$ are uniquely determined.

\section{Test Examples}

In the present section, three numerical test examples are given to explain the applicability of the proposed two techniques. Using own codes written in MATHEMATICA 10.0. package, the numerical results and figures are presented, as shown in the illustrative comparison tables.

The absolute error is given to compare the efficiency of the proposed schemes, given by $e_{N}=\left|f_{\text {Exact }}^{i}-f_{\text {Approximate }}^{i}\right|$, and evaluated at selected points for some $N$. The error norms $L_{2}$ and $L_{\infty}$ calculated in an interval $x \in[0, b]$ are given by

$$
L_{b}^{2}=\sqrt{h \sum_{i}^{l}\left(f_{\text {Exact }}^{i}-f_{\text {Approximate }}^{i}\right)^{2}},
$$

$$
L_{b}^{\infty}=\operatorname{Max}\left|f_{\text {Exact }}^{i}-f_{\text {Approximate }}^{i}\right|,
$$

for the $h$ step size along the interval $x \in[0, b]$. All numeric calculations are carried out on a regular machine Intel(R) Core(TM) i7 CPU, 3.2 GHz.

Example 1. Let us assume the following fourth-order boundary value problem

$$
\begin{gathered}
f^{(4)}(x)-\frac{1}{4}(1+x)^{-2} f^{\prime \prime}(x)+\frac{1}{2}(1+x)^{-4} f(x)=\frac{x^{2}-238 x+713}{(x+1)^{6}}, \\
x \in[0, \infty)
\end{gathered}
$$

with $f(0)=0, f^{\prime}(1)=1 / 2, f^{\prime \prime}(1)=1 / 2, f(x) \longrightarrow 2$ when $x$ $\longrightarrow \infty$.

We have

$$
\begin{gathered}
m=4, Q_{0}(x)=\frac{1}{2(1+x)^{4}}, Q_{1}(x)=0, Q_{2}(x)=-\frac{1}{4(1+x)^{2}}, \\
Q_{3}(x)=0, Q_{4}(x)=1, g(x)=\frac{x^{2}-238 x+713}{(x+1)^{6}} .
\end{gathered}
$$

Thus, for $N=5$, the numeric collocation points according to (35) are

$$
\begin{gathered}
x_{1}=\frac{1+1 / 4(1+\sqrt{5})}{1-1 / 4(1+\sqrt{5})}, \\
x_{2}=\frac{1-1 / 4(1-\sqrt{5})}{1+1 / 4(1-\sqrt{5})}, \\
x_{3}=\frac{1+1 / 4(1-\sqrt{5})}{1-1 / 4(1-\sqrt{5})}, \\
x_{4}=\frac{1-1 / 4(1+\sqrt{5})}{1+1 / 4(1+\sqrt{5})}, \\
x_{0} \longrightarrow \infty, x_{5}=0 .
\end{gathered}
$$
is

The fundamental matrix equation of problem using RRC

$\left\{\mathbf{Q}_{0} \mathbf{R}+\mathbf{Q}_{1} \mathbf{R} \mathbf{D}^{T}+\mathbf{Q}_{2} \mathbf{R}\left(\mathbf{D}^{T}\right)^{2}+\mathbf{Q}_{3} \mathbf{R}\left(\mathbf{D}^{T}\right)^{3}+\mathbf{Q}_{4} \mathbf{R}\left(\mathbf{D}^{T}\right)^{4}\right\} \mathbf{A}=\mathbf{G}$, 
while the fundamental matrix equation of problem using IRC is

$$
\begin{aligned}
& \left\{\mathbf{Q}_{0} \mathbf{R}+\mathbf{Q}_{1}\left(\mathbf{R D}^{T}+\mathbf{Z}\right)+\mathbf{Q}_{2}\left(\mathbf{R}\left(\mathbf{D}^{T}\right)^{2}+\mathbf{Z D}^{T}\right)\right. \\
& \quad+\mathbf{Z}^{\prime}+\mathbf{Q}_{3}\left(\mathbf{R}\left(\mathbf{D}^{T}\right)^{3}+\mathbf{Z}\left(\mathbf{D}^{T}\right)^{2}+\mathbf{Z}^{\prime} \mathbf{D}^{T}+\mathbf{Z}^{\prime \prime}\right) \\
& \left.\quad+\mathbf{Q}_{4}\left(\mathbf{R}\left(\mathbf{D}^{T}\right)^{4}+\mathbf{Z}\left(\mathbf{D}^{T}\right)^{3}+\mathbf{Z}^{\prime}\left(\mathbf{D}^{T}\right)^{2}+\mathbf{Z}^{\prime \prime} \mathbf{D}^{T}+\mathbf{Z}^{\prime \prime \prime}\right)\right\} \mathbf{A}=\mathbf{G}
\end{aligned}
$$

where $\mathbf{Q}_{0}, \mathbf{Q}_{2}, \mathbf{Q}_{4}, \mathbf{R}, \mathbf{D}^{T}, \mathbf{Z}, \mathbf{Z}^{\prime}, \mathbf{Z}^{\prime \prime}$, and $\mathbf{Z}^{\prime \prime \prime}$ are matrices with a given size of $6 \times 6$, for this example at $N=5$,

$\mathbf{Q}_{0}=\left[\begin{array}{cccccc}0 & 0 & 0 & 0 & 0 & 0 \\ 0 & 0.0000415747 & 0 & 0 & 0 & 0 \\ 0 & 0 & 0.00712393 & 0 & 0 & 0 \\ 0 & 0 & 0 & 0.0917553 & 0 & 0 \\ 0 & 0 & 0 & 0 & 0.334673 & 0 \\ 0 & 0 & 0 & 0 & 0 & 0.5\end{array}\right]$,

$$
\mathbf{Q}_{4}=\left[\begin{array}{llllll}
1 & 0 & 0 & 0 & 0 & 0 \\
0 & 1 & 0 & 0 & 0 & 0 \\
0 & 0 & 1 & 0 & 0 & 0 \\
0 & 0 & 0 & 1 & 0 & 0 \\
0 & 0 & 0 & 0 & 1 & 0 \\
0 & 0 & 0 & 0 & 0 & 1
\end{array}\right]
$$

$\mathbf{Q}_{2}=\left[\begin{array}{cccccc}0 & 0 & 0 & 0 & 0 & 0 \\ 0 & -0.00227966 & 0 & 0 & 0 & 0 \\ 0 & 0 & -0.0298411 & 0 & 0 & 0 \\ 0 & 0 & 0 & -0.107095 & 0 & 0 \\ 0 & 0 & 0 & 0 & -0.204534 & 0 \\ 0 & 0 & 0 & 0 & 0 & -0.25\end{array}\right]$,

$$
\mathbf{D}^{T}=\left[\begin{array}{cccccc}
0 & 3 / 4 & -2 & 3 & -4 & 5 \\
0 & -1 & 7 / 2 & -6 & 8 & 10 \\
0 & 1 / 4 & -2 & 21 / 4 & -8 & 10 \\
0 & 0 & 1 / 2 & -3 & 7 & -10 \\
0 & 0 & 0 & 3 / 4 & -4 & 35 / 4 \\
0 & 0 & 0 & 0 & 1 & -5
\end{array}\right]
$$

$\mathbf{R}=\left[\begin{array}{cccccc}1 & 1 & 1 & 1 & 1 & 1 \\ 1 & \frac{1}{4}(1+\sqrt{5}) & \frac{1}{4}(-1+\sqrt{5}) & \frac{1}{4}(1-\sqrt{5}) & \frac{1}{4}(-1-\sqrt{5}) & -1 \\ 1 & \frac{1}{4}(-1+\sqrt{5}) & \frac{1}{4}(-1-\sqrt{5}) & \frac{1}{4}(-1-\sqrt{5}) & \frac{1}{4}(-1+\sqrt{5}) & 1 \\ 1 & \frac{1}{4}(1-\sqrt{5}) & \frac{1}{4}(-1-\sqrt{5}) & \frac{1}{4}(1+\sqrt{5}) & \frac{1}{4}(-1+\sqrt{5}) & -1 \\ 1 & \frac{1}{4}(-1-\sqrt{5}) & \frac{1}{4}(-1+\sqrt{5}) & \frac{1}{4}(-1+\sqrt{5}) & \frac{1}{4}(-1-\sqrt{5}) & 1 \\ 1 & -1 & 1 & -1 & 1 & -1\end{array}\right]$,
TABLE 1: Comparing the CPU time used by seconds for RRC and IRC schemes.

\begin{tabular}{ccc}
\hline$N$ & $\begin{array}{c}\text { CPU time used by RRC } \\
\text { scheme }\end{array}$ & $\begin{array}{c}\text { CPU time used by IRC } \\
\text { scheme }\end{array}$ \\
\hline 4 & 0.094 & 0.155 \\
5 & 0.11 & 0.19 \\
6 & 0.156 & 0.241 \\
\hline
\end{tabular}

$$
\begin{aligned}
& \mathbf{Z}=\left[\begin{array}{cccccc}
0 & 0 & 0 & 0 & 0 & \frac{5}{4} \\
0 & 0 & 0 & 0 & 0 & \frac{5}{16}(-1-\sqrt{5}) \\
0 & 0 & 0 & 0 & 0 & \frac{5}{16}(-1+\sqrt{5}) \\
0 & 0 & 0 & 0 & 0 & \frac{5}{16}(-1+\sqrt{5}) \\
0 & 0 & 0 & 0 & 0 & \frac{5}{16}(-1-\sqrt{5}) \\
0 & 0 & 0 & 0 & 0 & \frac{5}{4}
\end{array}\right], \\
& \mathbf{Z}^{\prime}=\left[\begin{array}{cccccc}
0 & 0 & 0 & 0 & 0 & 0 \\
0 & 0 & 0 & 0 & 0 & \frac{15}{32}(-7+3 \sqrt{5}) \\
0 & 0 & 0 & 0 & 0 & \frac{-75}{32}(-3+\sqrt{5}) \\
0 & 0 & 0 & 0 & 0 & \frac{-15}{32}(7+3 \sqrt{5}) \\
0 & 0 & 0 & 0 & 0 & \frac{75}{32}(3+\sqrt{5}) \\
0 & 0 & 0 & 0 & 0 & -90
\end{array}\right], \\
& \mathbf{Z}^{\prime \prime}=\left[\begin{array}{cccccc}
0 & 0 & 0 & 0 & 0 & 0 \\
0 & 0 & 0 & 0 & 0 & \frac{15}{64}(-2+\sqrt{5}) \\
0 & 0 & 0 & 0 & 0 & \frac{75}{64}(90-41 \sqrt{5}) \\
0 & 0 & 0 & 0 & 0 & \frac{15}{64}(-2-\sqrt{5}) \\
0 & 0 & 0 & 0 & 0 & \frac{75}{64}(90+41 \sqrt{5}) \\
0 & 0 & 0 & 0 & 0 & 2280
\end{array}\right], \\
& \mathbf{Z}^{\prime \prime \prime}=\left[\begin{array}{cccccc}
0 & 0 & 0 & 0 & 0 & 0 \\
0 & 0 & 0 & 0 & 0 & \frac{15}{128}(901-403 \sqrt{5}) \\
0 & 0 & 0 & 0 & 0 & \frac{375}{128}(-1237-553 \sqrt{5}) \\
0 & 0 & 0 & 0 & 0 & \frac{15}{128}(901+403 \sqrt{5}) \\
0 & 0 & 0 & 0 & 0 & \frac{375}{128}(-1237-553 \sqrt{5}) \\
0 & 0 & 0 & 0 & 0 & -40020
\end{array}\right] .
\end{aligned}
$$


TABLE 2: Comparison between absolute errors with $\lambda=1$ given by two proposed schemes.

\begin{tabular}{lcccc}
\hline$x$ & & RRC scheme & & \multicolumn{2}{c}{ IRC scheme } \\
\hline 0 & $e_{20}$ & $e_{30}$ & $e_{20}$ & 1 \\
0.5 & 1 & 1 & $4.61847 \times 10^{-8}$ & $3.66704 \times 10^{-9}$ \\
1 & $2.23497 \times 10^{-7}$ & $1.59521 \times 10^{-9}$ & $5.93028 \times 10^{-7}$ & $6.13636 \times 10^{-9}$ \\
1.5 & $4.90784 \times 10^{-7}$ & $5.74586 \times 10^{-10}$ & $2.2526 \times 10^{-8}$ & $1.4928 \times 10^{-9}$ \\
2 & $4.68739 \times 10^{-9}$ & $2.52918 \times 10^{-9}$ & $1.8386 \times 10^{-6}$ & $9.67304 \times 10^{-9}$ \\
2.5 & $1.78495 \times 10^{-7}$ & $1.33078 \times 10^{-8}$ & $1.11684 \times 10^{-7}$ & $9.44468 \times 10^{-9}$ \\
3 & $1.43122 \times 10^{-6}$ & $1.99709 \times 10^{-8}$ & $2.11749 \times 10^{-6}$ & $2.75718 \times 10^{-9}$ \\
3.5 & $1.35397 \times 10^{-6}$ & $7.57517 \times 10^{-9}$ & $1.29148 \times 10^{-6}$ & $1.00546 \times 10^{-8}$ \\
5 & $2.16835 \times 10^{-6}$ & $3.82668 \times 10^{-8}$ & $1.27908 \times 10^{-6}$ & $2.26605 \times 10^{-8}$ \\
7.5 & $3.534 \times 10^{-6}$ & $2.72901 \times 10^{-8}$ & $2.23981 \times 10^{-7}$ & $6.70631 \times 10^{-8}$ \\
10 & $5.73755 \times 10^{-6}$ & $1.88112 \times 10^{-7}$ & $4.59453 \times 10^{-6}$ & $3.03553 \times 10^{-9}$ \\
\hline
\end{tabular}

TABLE 3: Comparison between absolute errors with $\lambda=2$ given by two proposed schemes.

\begin{tabular}{|c|c|c|c|c|}
\hline \multirow{2}{*}{$x$} & \multicolumn{2}{|c|}{ RRC scheme } & \multicolumn{2}{|c|}{ IRC scheme } \\
\hline & $e_{20}$ & $e_{30}$ & $e_{20}$ & $e_{30}$ \\
\hline 0 & 1 & 1 & 1 & 1 \\
\hline 0.5 & $3.31737 \times 10^{-6}$ & $3.91279 \times 10^{-8}$ & $4.85215 \times 10^{-6}$ & $2.86433 \times 10^{-7}$ \\
\hline 1 & $1.21648 \times 10^{-5}$ & $2.30618 \times 10^{-7}$ & $6.13597 \times 10^{-6}$ & $9.11931 \times 10^{-8}$ \\
\hline 1.5 & $1.68999 \times 10^{-5}$ & $3.20837 \times 10^{-7}$ & $2.99644 \times 10^{-5}$ & $6.97906 \times 10^{-8}$ \\
\hline 2 & $2.57922 \times 10^{-5}$ & $3.48555 \times 10^{-7}$ & $2.71531 \times 10^{-5}$ & $8.9908 \times 10^{-7}$ \\
\hline 2.5 & $2.07512 \times 10^{-5}$ & $4.35107 \times 10^{-7}$ & $8.88987 \times 10^{-6}$ & $1.01733 \times 10^{-7}$ \\
\hline 3 & $3.12895 \times 10^{-5}$ & $6.64248 \times 10^{-7}$ & $4.44485 \times 10^{-5}$ & $3.8419 \times 10^{-7}$ \\
\hline 3.5 & $1.99079 \times 10^{-5}$ & $2.69503 \times 10^{-7}$ & $1.5211 \times 10^{-5}$ & $1.08309 \times 10^{-6}$ \\
\hline 5 & $1.14161 \times 10^{-6}$ & $1.06864 \times 10^{-6}$ & $2.31729 \times 10^{-5}$ & $6.46631 \times 10^{-7}$ \\
\hline 7.5 & $5.4514 \times 10^{-5}$ & $1.43524 \times 10^{-6}$ & $2.57514 \times 10^{-5}$ & $1.97968 \times 10^{-7}$ \\
\hline 10 & $2.13571 \times 10^{-4}$ & $2.89479 \times 10^{-6}$ & $4.48328 \times 10^{-5}$ & $2.05962 \times 10^{-6}$ \\
\hline
\end{tabular}

Then, we obtain the augmented matrix (49) with respect to RRC as

$$
[\tilde{\mathbf{S}} ; \tilde{\mathbf{G}}]=\left[\begin{array}{cccccccc}
1 & -1 & 1 & -1 & 1 & -1 & ; & 0 \\
0 & 0.5 & 0 & -1.5 & 0 & 2.5 & ; & 0.5 \\
1 & 0.83333 & 0.38888 & -0.18518 & -0.69753 & -0.97736 & ; & 1.22222 \\
1 & 1 & 1 & 1 & 1 & 1 & ; & 2 \\
0.33467 & -28.72592 & 405.80168 & -2818.03109 & 12516.65380 & -33329.69198 & ; & 376.69653 \\
0.5 & -47.5 & 760.03125 & -6080.75 & 30513.0625 & -86383.3125 & ; & 713
\end{array}\right] .
$$

Then, we solve the equation (49) to find the RC coefficients in the matrix form:

$$
\mathbf{A}=\left[\begin{array}{llllll}
-0.0124202 & 1.00234 & 1.01636 & -0.00117103 & -0.00393679 & -0.00117103
\end{array}\right]
$$


TABLE 4: Comparing the $L_{10}^{2}$ and $L_{10}^{\infty}$ on interval $x \in[0,10]$ for $\lambda=1$.

\begin{tabular}{ccccc}
\hline$N$ & \multicolumn{2}{c}{ RRC scheme } & \multicolumn{3}{c}{ IRC scheme } \\
\hline 20 & $L_{10}^{2}$ & $L_{10}^{\infty}$ & $L_{10}^{2}$ & $4.59453 \times 10^{-6}$ \\
30 & $1.70683 \times 10^{-9}$ & $7.31028 \times 10^{-6}$ & $6.74223 \times 10^{-10}$ & $9.79285 \times 10^{-8}$ \\
\hline
\end{tabular}

TABLE 5: Comparing the $L_{10}^{2}$ and $L_{10}^{\infty}$ on interval $x \in[0,10]$ for $\lambda=2$.

\begin{tabular}{ccccc}
\hline$N$ & \multicolumn{2}{c}{ RRC scheme } & \multicolumn{2}{c}{ IRC scheme } \\
\hline 20 & $L_{10}^{2}$ & $L_{10}^{\infty}$ & $L_{10}^{2}$ & $L_{10}^{\infty}$ \\
30 & $1.45682 \times 10^{-6}$ & $2.13571 \times 10^{-4}$ & $6.41968 \times 10^{-8}$ & $5.10902 \times 10^{-5}$ \\
\hline
\end{tabular}

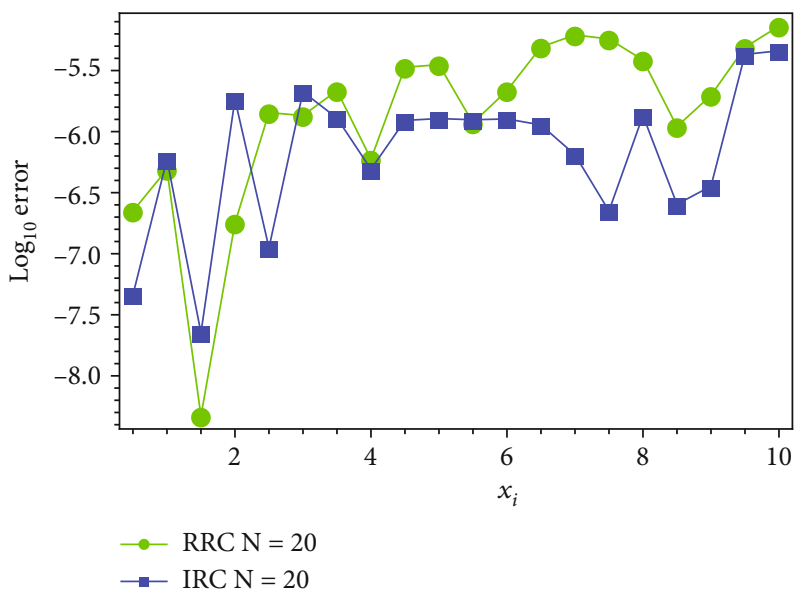

Figure 1: The absolute errors for the proposed two schemes when $N=20$ with $\lambda=1$.

TABLE 6: Comparing the CPU time used for RRC and IRC schemes.

\begin{tabular}{|c|c|c|c|c|}
\hline \multirow{2}{*}{$N$} & \multicolumn{2}{|c|}{ RRC scheme } & \multicolumn{2}{|c|}{ IRC scheme } \\
\hline & $\lambda=1$ & $\lambda=2$ & $\lambda=1$ & $\lambda=2$ \\
\hline 20 & 1.173 & 1.172 & 1.938 & 1.985 \\
\hline 30 & 3.89 & 3.858 & 4.591 & 4.61 \\
\hline
\end{tabular}

while the augmented matrix (49) with respect to IRC as

$$
[\tilde{S} ; \tilde{\mathbf{G}}]=\left[\begin{array}{cccccccc}
1 & -1 & 1 & -1 & 1 & -1 & ; & 0 \\
0 & 0.5 & 0 & -1.5 & 0 & 2.5 & ; & 0.5 \\
1 & 0.83333 & 0.38888 & -0.18518 & -0.69753 & -0.97736 & ; & 1.22222 \\
1 & 1 & 1 & 1 & 1 & 1 & 2 \\
0.33467 & -28.72592 & 405.42246 & -2799.72562 & 12543.47181 & -40972.42064 ; & ; 76.69653 \\
0.5 & -47.5 & 760.5 & -6159.5 & 33696.5 & -141135.5 & ; & 713
\end{array}\right] .
$$


TABLE 7: Comparison between absolute errors given by two proposed schemes.

\begin{tabular}{lcccc}
\hline$X$ & & RRC scheme & & \multicolumn{2}{c}{ IRC scheme } & $e_{20}$ \\
\hline 0 & $e_{20}$ & $e_{30}$ & 1 & \\
0.1 & 1 & 1 & $5.80324 \times 10^{-9}$ & $7.13895 \times 10^{-10}$ \\
0.7 & $2.20484 \times 10^{-8}$ & $4.0709 \times 10^{-9}$ & $1.84652 \times 10^{-8}$ & $6.01463 \times 10^{-8}$ \\
2.4 & $8.86658 \times 10^{-9}$ & $6.84228 \times 10^{-8}$ & $2.83721 \times 10^{-6}$ & $1.27556 \times 10^{-7}$ \\
3.8 & $5.81316 \times 10^{-7}$ & $2.90517 \times 10^{-7}$ & $2.68921 \times 10^{-6}$ & $2.70375 \times 10^{-8}$ \\
5 & $3.56672 \times 10^{-6}$ & $5.43355 \times 10^{-7}$ & $1.59222 \times 10^{-6}$ & $2.63469 \times 10^{-8}$ \\
6.7 & $4.19425 \times 10^{-6}$ & $6.64317 \times 10^{-7}$ & $4.96175 \times 10^{-6}$ & $5.53538 \times 10^{-8}$ \\
13.8 & $4.01577 \times 10^{-5}$ & $8.70798 \times 10^{-7}$ & $1.33586 \times 10^{-5}$ & $7.21039 \times 10^{-7}$ \\
14.4 & $1.06869 \times 10^{-4}$ & $9.22562 \times 10^{-7}$ & $2.31729 \times 10^{-5}$ & $5.63274 \times 10^{-8}$ \\
18.6 & $8.5269 \times 10^{-4}$ & $8.87894 \times 10^{-7}$ & $1.20205 \times 10^{-5}$ & $1.12601 \times 10^{-6}$ \\
20 & $1.26741 \times 10^{-4}$ & $1.59984 \times 10^{-6}$ & $1.20881 \times 10^{-4}$ & $9.73054 \times 10^{-7}$ \\
\hline
\end{tabular}

TABLE 8: Comparing the $L_{10}^{2}$ and $L_{10}^{\infty}$ on interval $x \in[0,10]$ for $\lambda=-1$.

\begin{tabular}{lccrr}
\hline$N$ & \multicolumn{2}{c}{ RRC scheme } & \multicolumn{2}{c}{ IRC scheme } \\
\hline 20 & $L_{10}^{2}$ & $L_{10}^{\infty}$ & $L_{10}^{2}$ & $1.20881 \times 10^{-4}$ \\
30 & $1.12301 \times 10^{-6}$ & $8.5269 \times 10^{-4}$ & $4.667 \times 10^{-7}$ & $2.83766 \times 10^{-6}$ \\
\hline
\end{tabular}
form:

Similarly, we compute the RC coefficients in the matrix

$$
\mathbf{A}=\left[\begin{array}{llllll}
0 & 1 & 1 & 0 & 0 & 0
\end{array}\right]
$$

Substituting by the RC coefficients of the RRC scheme in Equation (32), we get an approximate solution of this problem, while, if we use the RC coefficients of the IRC scheme in Equation (32), we get the exact solution $2 x(x-3) /(x+1)^{2}$. We note that the RRC scheme gives the exact solution at $N=6$ while the IRC scheme gives the exact solution at $N$ $=4$, which shows that the IRC scheme obtains better accuracy than the $\mathrm{RRC}$ due to the truncation found in $\mathrm{RRC}$ (18). In addition, the CPU time used for the RRC and IRC schemes at different Nis listed in Table 1. Table 1 shows that the time used for calculating the solution by RRC is less than the time for IRC, because of the added truncated terms in coding. Therefore, the time for getting the exact solution by IRC (better accuracy at $N=4$ ) is close to that given by RRC (at $N=6$ ). The following examples show the comparison of the two schemes strongly with numeric table's results.

Example 2. Consider Laguerre-eigen problem [26]

$$
x f^{\prime \prime}(x)+(x+1) f^{\prime}(x)+\lambda f(x)=0, \quad x \in[0, \infty),
$$

TABLE 9: Comparing the CPU time used by seconds for RRC and IRC schemes $\lambda=-1$.

\begin{tabular}{ccc}
\hline$N$ & $\begin{array}{c}\text { CPU time used by RRC } \\
\text { scheme }\end{array}$ & $\begin{array}{c}\text { CPU time used by IRC } \\
\text { scheme }\end{array}$ \\
\hline 20 & 0.844 & 0.971 \\
30 & 2.345 & 2.657 \\
\hline
\end{tabular}

has the exact eigen solutions

$$
\begin{gathered}
f(x)=1, \quad \lambda=0, \\
f(x)=e^{-x} L_{n}(x), \quad \lambda=n+1, n \geq 0,
\end{gathered}
$$

where $L_{n}(x)$ are the well-known Laguerre polynomials of degree $n$; Equation (66) is a boundary value problem, so the conditions are "natural" at both endpoints where $f(0)$ $=1$ and $f(x)=1$ when $x \longrightarrow \infty$. By applying the proposed two schemes as in the previous example, we obtain the solution $f(x)=1$, which is the exact eigen solution when $\lambda=0$. For $N=3$, the RRC scheme gives the exact eigen solution, while the IRC is satisfied with $N=2$ to find this solution. In Tables 2 and 3, the resulting values for $N=20$ and 30 using the present two schemes together with the exact values of the solution $f(x)=e^{-x} L_{n}(x), \lambda=n+1, n \geq 0$, are tabulated with different values of $\lambda$. The error reduces when the series increased. The numeric values of the error norms $L_{2}$ and $L_{\mathrm{\infty}}$ in interval $x \in[0,10]$ is given in Tables 4 and 5 with different values of $\lambda$. Additionally, the absolute errors $e_{N}$ for the two 
schemes when $N=20$ are plotted in Figure 1. In Table 6, the CPU time used for RRC and IRC schemes at different $N$ shows that IRC needs time more than RRC because of the calculation of the added terms of $B$.

Example 3. Consider Whittaker's equation eigen problem [26] of the form

$$
f^{\prime \prime}(x)+\left[\frac{-1}{4}+\frac{1}{f(x)}+\frac{\lambda}{f(x)}\right] f(x)=0, \quad x \in[0, \infty)
$$

where $\lambda$ is the eigen value; it represents a special case of Whittaker's equation. The exact given solution of (68) is $f($ $x)=e^{-0.5 x} L_{n}^{1}(x)$, where $\lambda=n, n \geq 0$, is an integer and $L_{n}^{k}(x)$ is the well-known associated Laguerre polynomials of $k^{\text {th }}$ order and degree $n$. If $\lambda=-1$, (68) gives a special case or linear Whittaker's equation as

$$
4 f^{\prime \prime}(x)-f(x)=0, \quad x \in[0, \infty) .
$$

The exact solution is $f(x)=e^{-0.5 x}$ with the boundary conditions $f(0)=1$ and $f(x)=1$ when $x \longrightarrow \infty$.

By the same procedure, the RC collocation method using the proposed two schemes is applied to solve (69) with the subjected boundary conditions. In Table 7 , the numerical result for $N=20$ and 30 using the proposed schemes is compared with the exact values of $f(x)=e^{-0.5 x}$. The computation of $L_{2}$ and $L_{\mathrm{o}}$ on interval $x \in[0,10]$ is given in Table 8. In Table 9, the CPU time used for RRC and IRC schemes at different $N$ shows that IRC needs time more than RRC.

\section{Conclusion}

A rational Chebyshev (RC) spectral collocation technique is considered in this paper to solve high-order ordinary differential equations (ODEs) defined on a semi-infinite domain using the proposed two schemes. Two definitions of the derivative of the RC functions are introduced, namely, the regular and improved definitions. Due to the two definitions, two schemes are presented for solving the proposed ODEs with variable coefficients in the semi-infinite interval. According to the convergence of the RC functions at the infinity, the proposed technique deals with the boundary value problem which is defined on semi-infinite domains easily. Furthermore, an intriguing advantage of this approach is the ability to find the analytical exact solutions if the equation has a solution in a rational function form. To demonstrate the applicability of the proposed approach, three illustrative examples are given. The calculated numerical values and comparisons proved that the improved scheme is better with more calculation than the regular scheme which is based on the truncation in the definition. The method may extend to the case of nonlinear DEs with variable coefficients, which the authors are investigating.

\section{Data Availability}

The authors declare that there is no data associated with this research.

\section{Conflicts of Interest}

The authors declare that they have no competing interests.

\section{Authors' Contributions}

All authors carried out the proofs and conceived of the study. All authors read and approved of the final form of the manuscript.

\section{Acknowledgments}

The researchers would like to thank the Deanship of Scientific Research, Qassim University, for funding the publication of this project.

\section{References}

[1] P. Agarwal, M. Attary, M. Maghasedi, and P. Kumam, "Solving higher-order boundary and initial value problems via Chebyshev-spectral method: application in elastic foundation," Symmetry, vol. 12, no. 6, p. 987, 2020.

[2] A. Akyüz and M. Sezer, "Chebyshev polynomial solutions of systems of high-order linear differential equations with variable coefficients," Applied Mathematics and Computation, vol. 144, no. 2-3, pp. 237-247, 2003.

[3] S. H. Aziz, M. Rasheed, and S. Shihab, "New properties of modified second kind Chebyshev polynomials," Journal of Southwest Jiaotong University, vol. 55, no. 3, p. 3, 2020.

[4] E. A. Butcher and O. A. Bobrenkov, "On the Chebyshev spectral continuous time approximation for constant and periodic delay differential equations," Communications in Nonlinear Science and Numerical Simulation, vol. 16, no. 3, pp. 15411554, 2011.

[5] V. N. Mishra, H. R. Marasi, H. Shabanian, and M. Nosraty, "Solution of Voltra-Fredholm integro-differential equations using Chebyshev collocation method," Global Journal of Technology and Optimization, vol. 8, no. 1, p. 210, 2017.

[6] A. H. Khater, R. S. Temsah, and M. Hassan, "A Chebyshev spectral collocation method for solving Burgers'-type equations," Journal of Computational and Applied Mathematics, vol. 222, no. 2, pp. 333-350, 2008.

[7] S. D. Kim and S. V. Parter, "Preconditioning Chebyshev spectral collocation method for elliptic partial differential equations," SIAM Journal on Numerical Analysis, vol. 33, no. 6, pp. 2375-2400, 1996.

[8] J. C. Mason and D. C. Handscomb, Chebyshev Polynomials, CRC press, 2002.

[9] M. Sezer and M. Kaynak, "Chebyshev polynomial solutions of linear differential equations," International Journal of Mathematical Education in Science and Technology, vol. 27, no. 4, pp. 607-618, 1996.

[10] S. Deniz and M. Sezer, "Rational Chebyshev collocation method for solving nonlinear heat transfer equations," International Communications in Heat and Mass Transfer, vol. 114, article 104595, 2020. 
[11] P. S. Malachivskyy, Y. V. Pizyur, and R. P. Malachivsky, "Chebyshev approximation by a rational expression for functions of many variables," Cybernetics and Systems Analysis, vol. 56, no. 5, pp. 811-819, 2020.

[12] X. Zhang and J. P. Boyd, "Revisiting the Thomas-Fermi equation: accelerating rational Chebyshev series through coordinate transformations," Applied Numerical Mathematics, vol. 135, pp. 186-205, 2019.

[13] S. Abbasbandy, H. Roohani Ghehsareh, and I. Hashim, "An approximate solution of the MHD flow over a non-linear stretching sheet by rational Chebyshev collocation method," UPB Scientific Bulletin, vol. 74, 2012.

[14] M. A. Ramadan, K. R. Raslan, T. S. El Danaf, and M. A. Abd El Salam, "On the exponential Chebyshev approximation in unbounded domains: a comparison study for solving highorder ordinary differential equations," International Journal of Pure and Applied Mathematics, vol. 105, no. 3, pp. 399413, 2015.

[15] M. A. Ramadan, K. R. Raslan, and M. A. Nassar, "An approximate analytical solution of higher-order linear differential equations with variable coefficients using improved rational Chebyshev collocation method," Applied and Computational Mathematics, vol. 3, no. 6, pp. 315-322, 2014.

[16] M. A. Ramadan, K. R. Raslan, and M. A. Nassar, "Numerical solution of system of higher order linear ordinary differential equations with variable coefficients using two proposed schemes for rational Chebyshev functions," Global Journal of Mathematics, vol. 3, no. 2, pp. 322-337, 2016.

[17] S. Yalçınbas, N. Özsoy, and M. Sezer, “Approximate solution of higher order linear differential equations by means of a new rational Chebyshev collocation method," Mathematical and Computational Applications, vol. 15, no. 1, pp. 45-56, 2010.

[18] K. Parand and M. Razzaghi, "Rational Chebyshev tau method for solving higher-order ordinary differential equations," International Journal of Computer Mathematics, vol. 81, no. 1, pp. 73-80, 2004.

[19] K. Parand and M. Razzaghi, "Rational Chebyshev tau method for solving Volterra's population model," Applied Mathematics and Computation, vol. 149, no. 3, pp. 893-900, 2004.

[20] K. Parand, Z. Delafkar, and F. Baharifard, "Rational Chebyshev tau method for solving natural convection of Darcian fluid about a vertical full cone embedded in porous media whit a prescribed wall temperature," World Academy of Science, Engineering and Technology, vol. 5, no. 8, pp. 1186-1191, 2011.

[21] A. M. Ramadan, K. R. Raslan, and M. A. Nassar, "Solving natural convection of Darcian fluid about a vertical full cone embedded in porous media whit a prescribed wall temperature is introduced using rational," Applied Mathematics and Information Science, vol. 14, no. 5, pp. 1-8, 2020.

[22] A. M. Ramadan, K. Raslan, and M. A. E. G. Nassear, "A rational Chebyshev functions approach for Fredholm-Volterra integro-differential equations," Computational Methods for Differential Equations, vol. 3, no. 4, pp. 284-297, 2015.

[23] M. A. Ramadan, K. Raslan, A. Hadhoud, and M. Nassar, "Numerical solution of high-order linear integro differential equations with variable coefficients using two proposed schemes for rational Chebyshev functions," New trends in mathematical sciences, vol. 4, no. 3, p. 22, 2016.

[24] A. M. Ramadan, D. Baleanu, and M. A.-G. Nassar, "Highly accurate numerical technique for population models via ratio- nal Chebyshev collocation method," Mathematics, vol. 7, no. 10, p. 913, 2019.

[25] B. Guo, J. Shen, and Z. Wang, "Chebyshev rational spectral and pseudospectral methods on a semi-infinite interval," International Journal for Numerical Methods in Engineering, vol. 53, no. 1, pp. 65-84, 2002.

[26] M. A. Ramadan, K. R. Raslan, A. R. Hadhoud, and M. A. Nassar, "Rational Chebyshev functions with new collocation points in semi-infinite domains for solving higher-order linear ordinary differential equations," Journal of Advances in Mathematics, vol. 11, no. 7, pp. 5403-5410, 2015. 